\title{
Nasal drug delivery
}

\author{
Elka Touitou $\cdot$ Lisbeth Illum
}

Published online: 8 November 2012

(C) Controlled Release Society 2012

The nasal cavity has for more than three decades been widely explored as a potential alternative route to oral or parenteral administration for systemically active drugs. The nasal route has shown remarkable advantages that include a rapid and high systemic availability, avoidance of first pass metabolism by the liver, and the possibility of targeting drugs directly from the nasal cavity to the brain $[1,2]$.

Considerable knowhow and data have been accumulated over the years from investigational work carried out by various excellent research groups in academia and industry. New nasal delivery carriers and emerging technologies have been used for product design and efficient clinical translation to nasal medicines.

The nasal route for systemic administration is attractive in many therapeutic areas where a rapid onset of action is required, e.g., pain, erectile dysfunction, frigidity, migraine, seizures, insomnia, panic attacks, Parkinson rigidity, hot flushes, emesis, Alzheimer or MS attacks, and cardiovascular events. An increasing number of small molecules are being marketed as nasal products such as Zomig $^{\circledR}$ (zolmitriptan), Imitrex ${ }^{\circledR}$ (sumatriptan), and Stadol NS (butorphanol tartrate) for migraine treatment, Aerodiol ${ }^{\circledR}$ (estradiol hemihydrate) for menopausal syndrome treatment, PecFent ${ }^{\circledR}$ and Instanyl ${ }^{\circledR}$ (fentanyl) for severe pain treatment, and Nicorette ${ }^{\circledR}$ (nicotine) for smoking cessation [3]. With this growing number of applications, the US market of intranasal

\footnotetext{
E. Touitou $(\bowtie)$

Institute for Drug Research, School of Pharmacy,

The Hebrew University of Jerusalem,

Jerusalem 91120, Israel

e-mail: touitou@cc.huji.ac.il

L. Illum

IDentity,

19 Cavendish Crescent North, The Park,

Nottingham NG7 1BA, UK

e-mail: lisbeth.illum@illumdavis.com
}

drug products is expected to reach US $\$ 5.2$ billion by 2017 [4]. It is noteworthy that most of the marketed products are based on molecules sufficiently lipophilic to enable therapeutic levels of the drug to reach the systemic circulation, thus requiring no nasal absorption enhancers.

Despite the obvious advantages of intranasal drug delivery, the nasal cavity presents a number of limitations for drug absorption, including low intrinsic permeability for some drugs, such as hydrophilic molecules, peptides, proteins, and nucleotides, rapid mucociliary clearance, and enzymatic degradation $[1,2]$.

In order to achieve efficient and safe intranasal drug products, a number of strategies for overcoming nasal delivery barriers can be applied. In the design of a nasal product, three main cooperative entities should be taken in consideration: the drug, the delivery carrier, and the administration device. The factors to be considered in the design and development of an efficient nasal product, related to these three components, are schematically presented as a three-lobe fleur-de-lys in Fig. 1.

Several important morphological and physiological constraints on nasal drug delivery including limited volume of administration requiring high drug potency or mucosal enzymatic drug degradation should be kept in mind when formulating new nasal products. Also, the key properties required from drug candidates for development of successful intranasal products would be potency, lipophilicity, and water solubility. The nasal delivery of hydrophilic or high molecular weight drugs would be in need of a nasal absorption promoter in order for the drug to be transported across the nasal membrane in sufficient quantity for therapeutic use.

Innovative strategy approaches to design efficient nasal delivery systems for specific drugs are currently in various stages of research and development. These include new nasal enhanced delivery technologies, design of carriers that impede drug degradation by mucosal enzymes, modulation 
Fig. 1 Three-lobe fleur-de-lys presentation of the factors to be considered in the design and development of an efficient nasal product

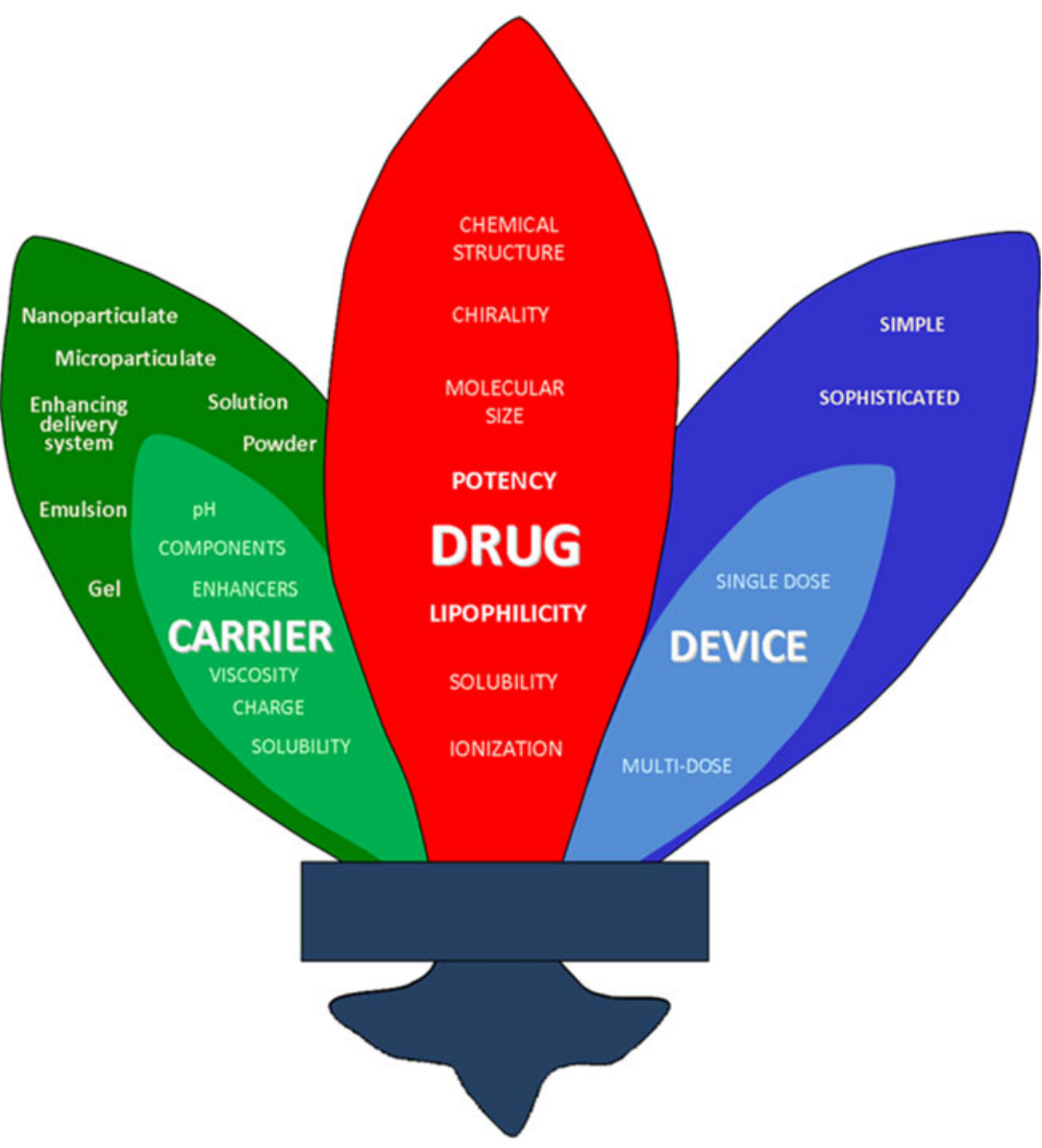

of carrier's physicochemical properties, and sophisticated devices for targeted drug delivery to brain. A recent review by Illum focuses on absorption enhancement technologies being developed commercially by companies specializing in nasal drug delivery [3].

In the delivery of peptides and proteins to the systemic circulation, the nasal route has many potential advantages over other administration methods, including the oral. However, as can be seen from the performance of a number of products presently on the market, such as calcitonin $\left(\right.$ Miacalcin $^{\circledR}$, Novartis, Fortical $^{\circledR}$, Unigene), desmopressin (Desmospray ${ }^{\circledR}$, Ferring), buserelin (Supre$\operatorname{cur}^{\circledR}$ and Sanofi-Aventis), nafarelin (Synarel ${ }^{\circledR}$, Pharmacia), and oxytocin (Syntocinon ${ }^{\circledR}$, Unites Pharmacies UK), nasal absorption of peptides and proteins from simple solutions is normally below $1 \%$. Hence, there is a great demand for novel, safe, and efficient nasal absorption enhancers for improved transport of less potent drugs across the nasal membrane [3].

This theme issue consists of nine papers that discuss various aspects of intranasal drug administration, including factors involved in the design of a nasal drug product for actives of various physicochemical characteristics including small molecular weight drugs, peptides, proteins, nucleic acids, and vaccines and strategies used in overcoming low drug absorption from the nasal cavity. An interesting paper also gives a thorough overview of existing nasal delivery devices and their pros and cons.

The first paper is a review by Suman et al. on the morphology and physiology of the nasal cavity and mucosa. The potential pathways of nasal drug absorption and factors affecting deposition and delivery, such as drug and formulation properties, administration device, inhalation techniques and pathological changes, are discussed by the authors.

Maggio and Pillon provide in their review a synopsis of a new class of recently developed and patented transmucosal absorption promoters, alkylsaccharides, called Intravail ${ }^{\circledR}$. An important feature of the alkylsaccharides, as presented by the authors, is their ability to overcome the poor bioavailability of many macromolecules and the potential damage conferred to the nasal mucosa by previously explored absorption enhancers. The paper describes the chemical and pharmacological properties of these enhancers along with recent developments in their use in preclinical studies and human clinical trials. The use of these excipients in design of new intranasal formulations of peptidic and nonpeptidic drugs and their commercial implications are also explored.

Another currently available option for intranasal administration of proteins is the exploitation of a novel nasal delivery system based on Solutol HS15 (CriticalSorb ${ }^{\mathrm{TM}}$ ), 
developed by Lewis, Jordan, and Illum. The authors present data showing that CriticalSorb is able to overcome the poor intrinsic absorption of proteins across the nasal mucosa without causing the side effects associated with known permeation enhancers. Studies in various animal models and in man have shown that nasal administration of proteins by using this delivery system results in a significantly enhanced systemic delivery and pharmacodynamics similar to those seen after subcutaneous injection.

Powder-based mucoadhesive formulations are another delivery strategy widely studied for nasal drug delivery. Saladini and co-authors describe in their research paper, work carried out on powder-based formulations containing mucoadhesive chitosan/pectin polyelectrolyte complex microparticles for nasal administration of the antiAlzheimer drug, tacrine hydrochloride. In their study, the scientists focus on the preparation and characterization of chitosan/pectin polyelectrolyte complexes with different amounts of chitosan. The results of this study show that the complexes can be used in the formulation of mucoadhesive microparticles with different functional properties, such as water uptake, mucoadhesion behavior, and enhanced drug permeation at the administration site.

Djupesland reviews current and new devices for nasal drug delivery. The author first describes the nasal anatomy and physiology and their impact on intranasal drug delivery. The background section is followed by a thorough presentation of the existing and emerging nasal delivery devices and dispersion technologies with emphasis on bidirectional delivery. A special focus is given to their advantages and limitations, as well as clinical performance.

An important application area of the intranasal delivery is pain management. Studies have demonstrated that the intranasal route could be as efficient as the intravenous one in delivering analgesics. Several intranasal pain management medications have been developed for postoperative pain, chronic and acute pain, and migraines. Wermeling discusses the use of a naloxone nasal spray as a response to opioid overdose epidemic: following a background on nasal delivery, the author summarizes the properties of naloxone hydrochloride dehydrate and discusses the translation of intranasal opioid formulations to naloxone nasal spray. The paper further reviews the biopharmaceutics of intranasal naloxone and clinical experience with intranasal naloxone in reversing narcosis. In this review, important safety considerations for intranasal naloxone as well as gaps and awareness in the use of naloxone spray are emphasized.

Watts et al. review the nasal delivery of fentanyl from formulation to pharmacokinetic profiles emphasizing the use of nasal fentanyl in breakthrough cancer pain. The paper describes clinical experience with nasal fentanyl from early studies to clinical data on recent products and also discusses the use of nasal fentanyl in children.

The RNAi local application to the respiratory mucosa presents many advantages by avoiding serum nuclease degradation, nonspecific accumulation, and rapid renal clearance. A review on the clinical translation of RNAibased treatments for respiratory diseases is presented by Gonzales et al. In their paper, the authors outline the important steps in the clinical translation of RNAi-based respiratory therapies. They focus on the disease and RNA target selection, siRNA design, respiratory barriers, and delivery technology. The paper presents antiviral therapies and preclinical evaluation with focus on the current status of clinical trials. Attention is also given to biological and physiological barriers, safe siRNA design, and the essential requirements for translation of an RNAi-based technology to a feasible therapy.

Zaman et al. review vaccine formulations designed for delivery to the nasal lymphoid tissue and focus on the association of antigens with mucosal adjuvants and delivery systems. The review summarizes the advantages and disadvantages of nasal vaccination and discusses leading strategies in delivery systems and adjuvants currently under investigation for the development of nasal vaccines. The authors present the advantages of this noninvasive, needlefree route for vaccination programs, by enhancing patient compliance and reducing the need to be administered by specialized healthcare workers.

Acknowledgments We would like to thank all the contributors for their efforts and commitment in making this special issue possible. Special thanks to Prof. Labhasetwar for his support in editing this issue.

\section{References}

1. Lim ST, Forbes B, Brown MB, Martin GP. Physiological factors affecting nasal drug delivery. In: Touitou E, Barry BW, editors. Enhancement in drug delivery. Boca Raton: CRC Press; 2007.

2. Pillon DJ, Arnold JJ, Meezan E. Nasal delivery of peptides. In: Touitou E, Barry BW, editors. Enhancement in drug delivery. Boca Raton: CRC Press; 2007.

3. Illum L. Nasal drug delivery-recent developments and future prospects. J Control Release. 2012;161:254-63.

4. Expanding therapeutic applications to drive the US intranasal drug delivery market, according to new report by Global Industry Analysts. http://www.prweb.com/releases/intranasal_drug_delivery/ allergic_infections/prweb9490139.htm 